\title{
Evaluation de la diversité floristique en herbacées dans le Parc National de Manda au Tchad
}

\author{
Isaac NDOTAM TATILA ${ }^{1,3^{*}}$, Frédéric REOUNODJI ${ }^{2}$, Joseph LUMANDE KASALI $^{3}$ et \\ Jean DIAOUANGANA ${ }^{4}$ \\ ${ }^{I}$ Université de Sarh, Institut Universitaire des Sciences Agronomiques et de l'Environnement, \\ BP : 105, Sarh (Région du Moyen Chari), République du Tchad. \\ ${ }^{2}$ Université des Sciences et Technologie d'Ati, BP : 20, Ati (Région du Batha, République du Tchad. \\ ${ }^{3}$ Université de Kinshasa, Ecole Régionale Post-Universitaire d'Aménagement et de Gestion Intégrés des \\ Forêts et Territoires Tropicaux, BP : 15373, Kinshasa, République Démocratique du Congo. \\ ${ }^{4}$ Faculté des Sciences et Techniques, Université Marien Ngouabi de Brazzaville, Congo. \\ *Auteur correspondant ; E-mail : indotamtatila@yahoo.fr;ndotamtatila@gmail.com.
} Tel : (+235) $66418797 / 99385138$

\section{REMERCIEMENTS}

Nous remercions vivement le Programme d'Appui à la Conservation des Ecosystèmes du Bassin du Congo (PACEBCo) qui a financé ces travaux de recherche à travers l'Ecole Régionale Post-Universitaire d'Aménagement et de Gestion Intégrés des Forêts et Territoires Tropicaux (ERAIFT).

\section{RESUME}

Situé dans la partie méridionale du Tchad, le Parc National de Manda fait partie du biome "savanes et forêts claires soudaniennes" figurant parmi les 200 écorégions essentielles au monde pour la conservation de la biodiversité globale. La connaissance de la biodiversité surtout végétale étant á la base des aménagements durables de l'environnement, l'objectif de cette étude est d'apprécier la composition floristique et la qualité des herbages dans les différentes unités géomorphologiques du Parc National de Manda (PNM) au Tchad en vue de fournir des éléments de gestion et d'amélioration adaptés aux conditions actuelles. L'état de la végétation herbacée du PNM a été établi à travers la détermination de la composition floristique, du recouvrement et de la richesse floristique dans 3 types d'unités géomorphologiques (plateaux, zones mixtes et bas-fonds). Les relevés effectués par la méthode des points quadrats alignés ont permis d'identifier 137 espèces reparties en 94 genres et 33 familles. Les cinq principales familles, Poaceae, Fabaceae, Malvaceae, Cyperaceae et Rubiaceae représentent $66,42 \%$ du cortège végétal dont $34,29 \%$ pour les graminoïdes : Poaceae $(25,54 \%)$ et Cyperaceae $(8,75 \%)$. Le nombre de taxons herbacés recensés a été de 100 respectivement dans les relevés des plateaux et dans les relevés des bas-fonds et de 85 dans ceux zones mixtes. 58 espèces sont communes aux 3 unités géomorphologiques. Le recouvrement herbacé est globalement élevé $(99,73 \%)$ et varie peu entre les différentes unités géomorphologiques. L'indice de diversité de Shannon et l'indice de régularité de Piélou pour l'ensemble du parc sont respectivement de 5,25 bits et 0,74 . Il y a donc une diversité floristique élevée et les individus se répartissent équitablement entre les différentes espèces.

(C) 2017 International Formulae Group. All rights reserved.

Mots clés : Tchad, taxons, recouvrement, végétation herbacée, unité géomorphologique, indice de diversité. 


\section{INTRODUCTION}

Les aires protégées sont des espaces voués à la conservation des ressources naturelles. Elles sont porteuses de valeurs écologiques et environnementales et, constituent un réservoir important de biodiversité floristique et faunique. Elles présentent souvent une valeur patrimoniale. Aussi, représentent-elles un élément important d'aménagement du territoire car leur existence entraîne de nombreuses modifications dans les règles d'accès à l'espace.

Les inventaires floristiques et l'étude des groupements végétaux constituent l'une des plus importantes sources de données de base notamment pour des recherches portant sur la diversité spécifique, le développement de systèmes de gestion forestière, d'aménagement et de conservation de la nature (Masharabu, 2010). On ne peut pas alors prétendre faire de la gestion des réserves naturelles sans connaître la structure, la dynamique et le fonctionnement des phytocénoses.

Au Tchad, les premières connaissances botaniques ont commencé en 1869 avec Natchigal, en 1900 par la mission FourreauLamy puis Corti, Gillet, et Maire. Ces travaux ce sont poursuivis vers les années 1950 et après les indépendances par des explorations assez poussées dans le Tibesti (Nord) et dans la partie méridionale. Cependant, ces investigations n'ont pas couvert l'ensemble tout le pays (Melom, 2015). La richesse floristique du Tchad est estimée à 4318 espèces et celle de la zone soudanienne dont fait partie le PNM est à 2750 espèces (MERH, 2014).

Le Parc national de Manda (PNM) est l'une des plus anciennes aires protégées du Tchad. Situés dans la zone soudanienne, il constitue un sanctuaire de biodiversité : mammifères, oiseaux et végétation de savane... Sa végétation fait partie du biome "savanes et forêts claires soudaniennes" classé en danger par le Fonds Mondial pour la Nature, en anglais, World Wide Fund for Nature (WWF) et figurant parmi le 200 écorégions essentielles au monde pour la conservation de la biodiversité globale (Boulanodji, 2010 ; Ouya, 2010). Le PNM est également classé parmi les "Important Bird Area" (IBA) par BirdLife du fait qu'il comporte des espèces qui sont restreintes à des biomes particuliers et qui dépendent de la conservation de cette végétation pour leur survie (Worge, 2012). Cependant, cette aire protégée a été peu étudiée (Saradoum, 2012) et ses ressources naturelles sont mal connues.

L'objectif de ce travail est de d'apprécier la composition floristique et qualité des herbages dans les différentes unités géomorphologiques du PNM en vue de fournir des éléments de gestion et d'amélioration adaptés aux conditions actuelles. La présente étude est la contribution à la mise en place des bases de données biologiques et écosystémiques sur le PNM. Il s'agira plus spécifiquement d'analyser la végétation herbacée, la composition et la diversité floristique du parc. Cette approche permettra de mieux comprendre la richesse spécifique de la végétation herbacée du PNM.

\section{MATERIEL ET METHODES}

Localisation de la zone d'étude

Le PNM couvre actuellement une superficie de 114000 ha (Chardonnet et al., 2010). Il est situé, entre la latitude $9^{\circ} 20^{\prime}$ et 9 $\circ 35^{\prime}$ Nord et la longitude $17^{\circ} 45^{\prime}$ et $18^{\circ} 20^{\prime}$ Est, et distant de $25 \mathrm{~km}$ au Nord-Ouest de la ville de Sarh, capitale du département du Barh Kôh et de la région du Moyen Chari. Son altitude varie de $344 \mathrm{~m}$ à $691 \mathrm{~m}$ (Figure 1).

\section{Hydrographie}

Le PNM est situé exactement au centre $\mathrm{du}$ Bassin versant du Chari qui couvre tout le sud-est du Tchad. Il est délimité dans sa partie orientale par le fleuve Chari et au Sud par le Barh Sara. Deux des trois principaux affluents du Chari ont leurs confluents dans le PNM : le Bahr Sara et le Bahr Salamat. En outre, il est identifié 13 mares temporaires et 23 mares permanentes dans le parc (Kolmagne, 2000).

\section{Climat}

Situé dans la zone bioclimatique soudanienne, le PNM jouit d'un climat tropical humide à 2 saisons alternées: une saison sèche qui va de novembre à mars et une saison des pluies, d'avril à octobre. La 
pluviométrie moyenne annuelle est de 1061,41 mm. Les températures annuelles sont de l'ordre de $21{ }^{\circ} \mathrm{C}$ à $28{ }^{\circ} \mathrm{C}$ avec une humidité relative selon les mois de 32 à $85 \%$ (ASECNA de Sarh, 2015).

\section{Sols}

On distingue dans le PNM les différents types de sol suivants:

- Les sols d'érosion sur roches acides issus de l'érosion qui constituent le Mont Niellim ;

- Les sesquioxydes à tâches et concrétions ferrugineuses et cuirasses qui se rencontrent sur les dalles latéritiques dans la zone de Niellim ;

- Les vertisols dans certaines zones inondables entre Koutou et Niellim ;

- Les sols ferralitiques qui affleurent largement dans les zones de Koutou et de Djoli ;

- Les sols ferrugineux tropicaux au centre du parc, de Nguéré à Koutou ;

- Enfin les sols hydromorphes caractéristiques des sols du Sud (Pias, 1964)

Le PNM présente un paysage varié composé de cours d'eau (Chari, Bahr Sara), des mares, des plaines d'inondation sableuses, des bas plateaux et de collines. Son altitude varie de 362 à $452 \mathrm{~m}$ (Chardonnet et al., 2010).

\section{Méthodes}

Réalisation des relevés : Méthode des points quadrats alignés

La méthode consiste à recenser la présence des espèces herbacées à la verticale de 100 «points » positionnés sur une ficelle de $20 \mathrm{~m}$ de longueur graduée tous les $20 \mathrm{~cm}$ et tendu au-dessus du tapis herbacé. On déplace perpendiculairement au sol, le long de la ficelle, une tige métallique fine qui est chaque fois descendue jusqu'au sol. On enregistre toutes les espèces herbacées qui sont en contact avec la tige métallique. Plusieurs espèces peuvent être touchées la tige au même point de lecture mais chaque espèce n'est notée qu'une seule fois. Si en revanche aucun végétal ne touche la tige métallique on note la présence d'un sol nu.

Sur chaque parcelle élémentaire (placeau de $2500 \mathrm{~m}^{2}$ ), 100 points de lecture sont effectués sur les demi-diagonales, ce qui donne 400 points de lecture sur chacune des parcelles élémentaires (Figure 1).

\section{Traitement des données}

Les données recueillies sur les fiches de relevés sont saisies et traitées à l'aide du tableur Excel. Les descripteurs suivants sont déterminés comme suit :

la fréquence spécifique (Fsi) d'une espèce (i) qui représente la somme cumulée des contacts de cette espèce sur la ligne de lecture ;

- $\quad$ la contribution spécifique, notée : Csi $(\%)=(\mathrm{Fsi} / \Sigma \mathrm{Fsi}) * 100(1)$

Où :

Fsi est la fréquence spécifique de l'espèce $\mathrm{i}$ représentant la somme des contacts de cette espèce sur la ligne de lecture ;

$\sum$ Fsi est la somme de contact de toutes espèces ;

Csi est la contribution spécifique de l'espèce $\mathrm{i}$.

l'intervalle de confiance ou indice de confiance (IC) ou précision de mesures d'une observation donnée. Lorsque IC $\leq 5 \%$, on considère que l'effet du hasard est éliminé. L'intervalle de confiance (IC) ou indice de confiance est donné par la formule : IC $(\%)=$ $\pm 2 \sqrt{\mathrm{n}(\mathrm{N}-\mathrm{n})} / \mathrm{N}^{2}$

Où :

$\mathrm{N}$ est l'effectif cumulé des contacts de l'ensemble des espèces, n l'effectif cumulé des contacts de l'espèce dominante ;

- $\quad$ le recouvrement spécifique (Ri) est le rapport entre la fréquence spécifique de l'espèce (i) sur le nombre total des points de lecture du relevé (3).

le recouvrement global $(\mathrm{RG})$ a été calculé par la formule suivante : $\mathrm{RG}(\%)=((\mathrm{N}-\mathrm{ni}) / \mathrm{N}) * 100$ (4)

Où :

$\mathrm{N}$ est le nombre total de contact et ni le nombre de points où le sol est nu.

Plusieurs formules existent et permettent d'évaluer la ressemblance floristique entre des végétations. Parmi celles-ci, l'indice de similitude $(C s)$ de Sørensen ou diversité $\beta$ a été choisi. Il permet de quantifier le degré de ressemblance de deux listes d'espèces de deux sites $\mathrm{A}$ et $\mathrm{B}$. Dans cette formule, $a$ représente le nombre d'espèces du site $\mathrm{A}, b$ le nombre 
d'espèces du site $B$ et $c$ le nombre d'espèces communes aux deux sites A et B (Minda, 2016).

$$
C S=\frac{2 a}{2 a+b+c}(5)
$$

La structure spécifique des espèces a été analysée à l'aide des indices de diversité de Shannon et Weaver $(\mathrm{H})$ et de régularité de Piélou (E). Les formules utilisées pour calculer ces indices sont :

-

$$
\mathrm{H}=-\sum \mathrm{pi} \log _{2} \text { (pi) (6) }
$$

pi $=$ ni/ $\mathrm{N}$ est la proportion des individus dans l'échantillon total qui appartiennent à l'espèce $\mathrm{i}$; ni est le nombre d'individus d'une espèce $\mathrm{i}$; $\mathrm{N}$ est le nombre total d'individus dans le parcours ; $\log _{2}$ est le logarithme base 2 . H est l'indice de diversité de Shannon.

- $\quad \mathrm{E}=\mathrm{H} / \mathrm{Hmax}$ (7) Où :

- $\quad$ Hmax est égal à $\log _{2}(n i / N) / \sum F s i$

Ces deux indices sont évalués sur des relevés bruts dans le but d'apprécier le niveau d'organisation du peuplement.

L'indice de diversité de Shannon et Weaver est fondé sur la théorie de l'information. Cet indice varie à la fois en fonction du nombre d'espèce et de la proportion relative du recouvrement de différentes espèces. Il est exprimé en bits, les valeurs extrêmes sont comprises entre 0,5 et 4,5 bits pour des relevés de petite taille. Selon
Nshimba (2007) l'indice de diversité de Shannon et Weaver, peut être maximal (Hmax) en prenant des valeurs comprises entre 8 et 9 pour des échantillons comprenant 100 à 200 espèces.

On lui associe l'indice de régularité qui varie de 0 à 1 . E tend vers 0 lorsque la quasi-totalité des effectifs appartiennent à une seule espèce. $E$ tend vers 1 lorsque chacune des espèces est représentée par le même nombre d'individus.

L'indice de Margalef (I) Index, ou indice de Margalef biodiversité est une mesure utilisée dans l'écologie pour estimer la biodiversité d'une communauté basée sur la distribution numérique des individus d'espèces différentes en fonction du nombre total d'individus de l'échantillon. I se traduit par la formule suivante :

$$
I=\frac{(S-1)}{\operatorname{Ln}(N)}
$$

Où :

$\mathrm{I}=$ Indice de Margalef

$\mathrm{S}=$ Nombre d'espèces

$\mathrm{N}=$ Nombre d'individus

Les valeurs inférieures à 2,0 sont considérées pour désigner les zones de faible diversité (généralement à la suite d'effets anthropiques) et les valeurs supérieures ou égales à 5,0 ci-dessus sont considérées comme indicateur de haute biodiversité.

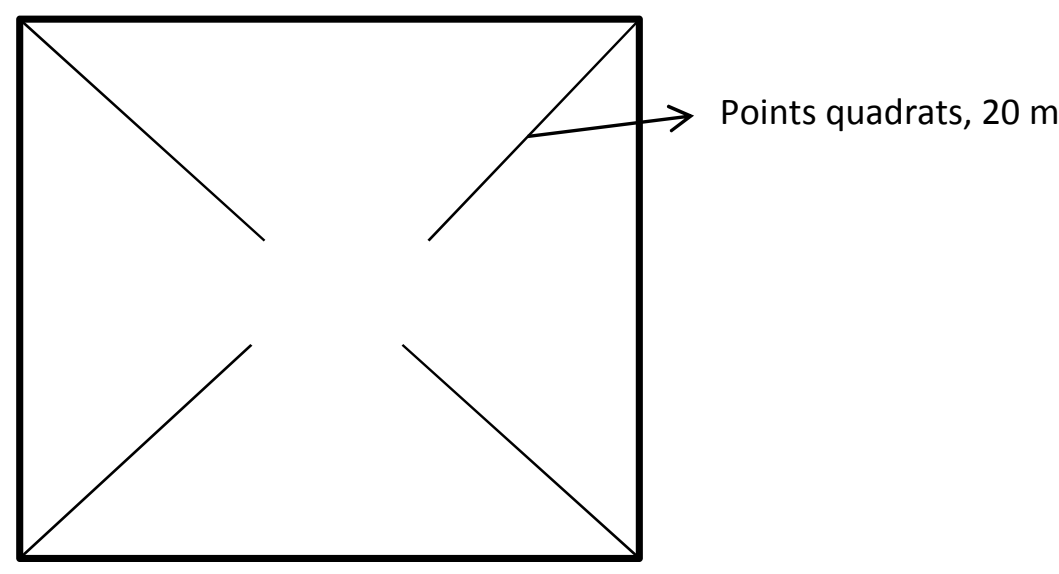

$50 \mathrm{~m}$

Figure 1: Schéma du dispositif de relevé de végétation. 


\section{RESULTATS}

La végétation herbacée de la zone du parc étudiée est riche de 137 espèces, reparties en 94 genres et 33 familles. Ces familles peuvent être regroupées en 4 lots :

un premier lot de 20 familles (Anthericaceae, Asparagaceae, Araceae, Bixaceae, Caryophyllaceae, Commelinaceae, Cucurbitaceae, Discoreceae, Hypoxidaceae, Iridaceae, Nympheacea, Onagraceae, Oxalidaceae, Pedaliaceae, Phyllanthaceae, Polygalaceae, Potulacaceae, Taccaceae, Vitaceae, Zingiberaceae) représentées chacune par un seul genre et une espèce ;

- un deuxième lot de 5 familles dont les Amaranthaceae, Apocynaceae et Euphorbiaceae comportant chacune 2 genres et 2 espèces, et, les Orobanchaceae avec chacune un genre et deux espèces ;

- un troisième lot composé de 6 familles dont les Malvaceae comportant 8 genres et 13 espèces, les Cyperaceae et les Rubiaceae comportant chacun 5 genres et respectivement 12 et 9 espèces, les Acanthaceae et les Lamiaceae comportant chacun 4 genres et respectivement 5 et 4 espèces, les Asteraceae comportant 4 genres et 4 espèces et les Convolvulaceae avec 3 genres et 4 espèces ;

un quatrième lot de 2 familles représentées par les Poaceae comportant 23 genres et 35 espèces et les Fabaceae avec 11 genres et 22 espèces (Figure 3 ) .

Les cinq principales familles, Poaceae,

Fabaceae, Malvaceae, Cyperaceae et Rubiaceae représentent $66,42 \%$ du cortège végétal dont $33,43 \%$ pour les graminoïdes : Poaceae (25,54\%) et Cyperaceae (8,75\%).

$$
\text { Trois types d'unités }
$$
géomorphologiques ont été identifiés. Ce sont.

- les plateaux à structure sablonneuse et sablo-limoneux. Ils sont composés des sols ferralitiques et ferralitiques ;
- les bas-fonds ou sols des dépressions à structure limono-argileuse. Ce sont des sols inondables et hydromorphes ;

- et les zones mixtes qui sont les intermédiaires entre les plateaux et les basfonds.

Au total 90 relevés ont été effectués, dont sur 29 les plateaux, 24 sur les zones mixtes et 37 sur les bas-fonds. Sur les 19967 individus recensés, quatre espèces totalisent une contribution spécifique de $39,48 \%$.Il s'agit de Hyperthelia dissoluta (12,46\%), Hypparenia rufa (10,33\%), Brachiaria kotschyana (8,73\%), Andropogon gayanus $(7,94 \%)$. Les autres espèces sont faiblement représentées, leur contribution spécifique est inférieure à $4 \%$.

L'indice de confiance calculé est de $0,66 \%$ pour l'ensemble de relevés. Il est de $0,72 \%$, pour les végétations des zones mixtes $0,62 \%$ pour celles des plateaux et des basfonds. Ces différents indices de confiances sont inférieurs à $5 \%$. Ainsi, l'effet du hasard est éliminé pour les différents relevés.

Le recouvrement herbacé est globalement élevé $(99,73 \%)$ pour l'ensemble de relevés et pour les différentes unités géomorphologiques $(99,30 \%)$ pour les plateaux, $99,94 \%$ pour les zones mixtes et $99,95 \%$ pour les bas-fonds).

Cependant, le recouvrement spécifique est généralement faible pour toutes les espèces (inférieur à $30 \%$ ) sur l'ensemble de relevés et sur les différentes unités géomorphologiques.

Selon le recouvrement spécifique, les espèces peuvent être classées en deux groupes. Le premier groupe comprend les espèces ayant un recouvrement supérieur à $10 \%$. Ce sont Hyperthelia dissoluta (27,65\%), Hyparrhenia rufa (22,93\%), Brachiaria kotschyana (19,38\%) et Andropogon gayanus $(17,52)$. Le deuxième groupe rassemble les espèces ayant un recouvrement inférieur à 10\%. Ce sont Oryza barthii (6,97\%), Andropogon africanus (6,63\%), Beckeropsis 
uniseta (5,87\%), Aspilia kotschyi (5,73\%), Paspalum scorbiculatum (5,68\%), Hyparrhenia bagirmica (5,03\%), Tephrosia linearis (4,66\%), Eragrotis cilianensis (4,37\%), Siphonochilus aethiopicus (4,27\%) et Andropogon schirensis (4,07\%).

Toutefois, la contribution des espèces abondantes est de 39,47\%.

Les degrés d'homogénéité spatiale et interne des espèces appréciés respectivement par les indices de Shannon, d'équitabilité et de Margalef pour tout l'ensemble de la zone d'étude et pour les trois unités géomorphologiques sont représentés dans le Tableau 1.

Le coefficient de similitude de Sorensen entre les différentes unités géomorphologiques est calculé. Cet indice est de $57,9 \%$ entre les plateaux et les zones mixtes, de 54,6\% entre les plateaux et les basfonds et de $56,6 \%$ entre les zones mixtes et les bas-fonds. (Tableau 2).

En effet, parmi les 137 espèces recensées, 58 espèces $(42,33 \%)$ sont communes aux 3 unités géomorphologiques, 33 espèces $(24,08 \%)$ sont présentes dans une seule unité et 46 espèces $(33,57 \%)$ au niveau de deux unités (Tableau 3).

$\mathrm{Au}$ total 137 espèces appartenant à 94 genres et 33 familles ont été recensées lors de notre étude. En effet, notre étude a permis d'identifier 23 nouvelles espèces, 6 nouveaux genres et une nouvelle famille. Ainsi, le parc national de Manda comprend 392 espèces recensées regroupées en 200 genres et 56 familles.
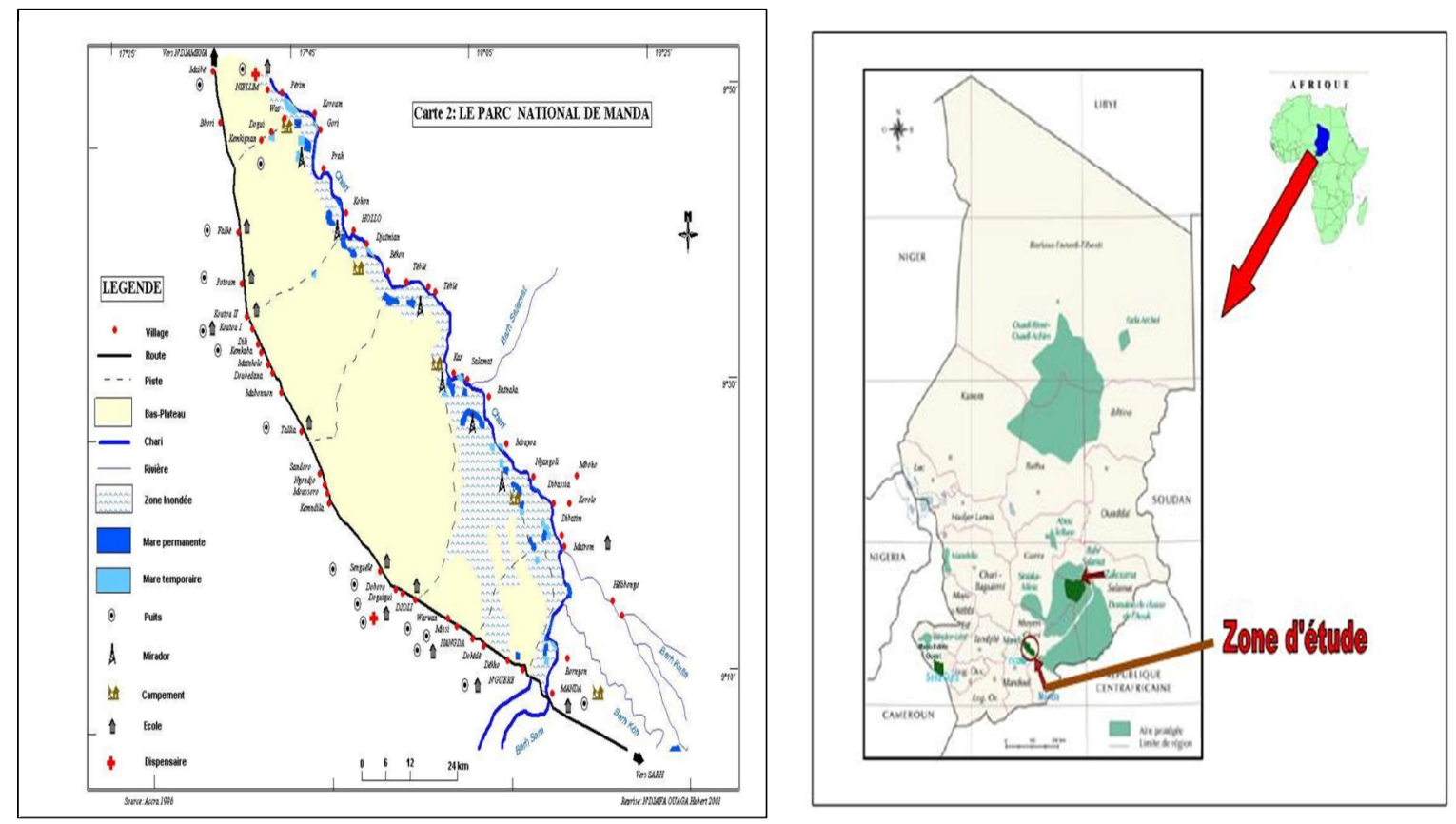

Figure 2 : Carte localisation du Parc National de Manda. 


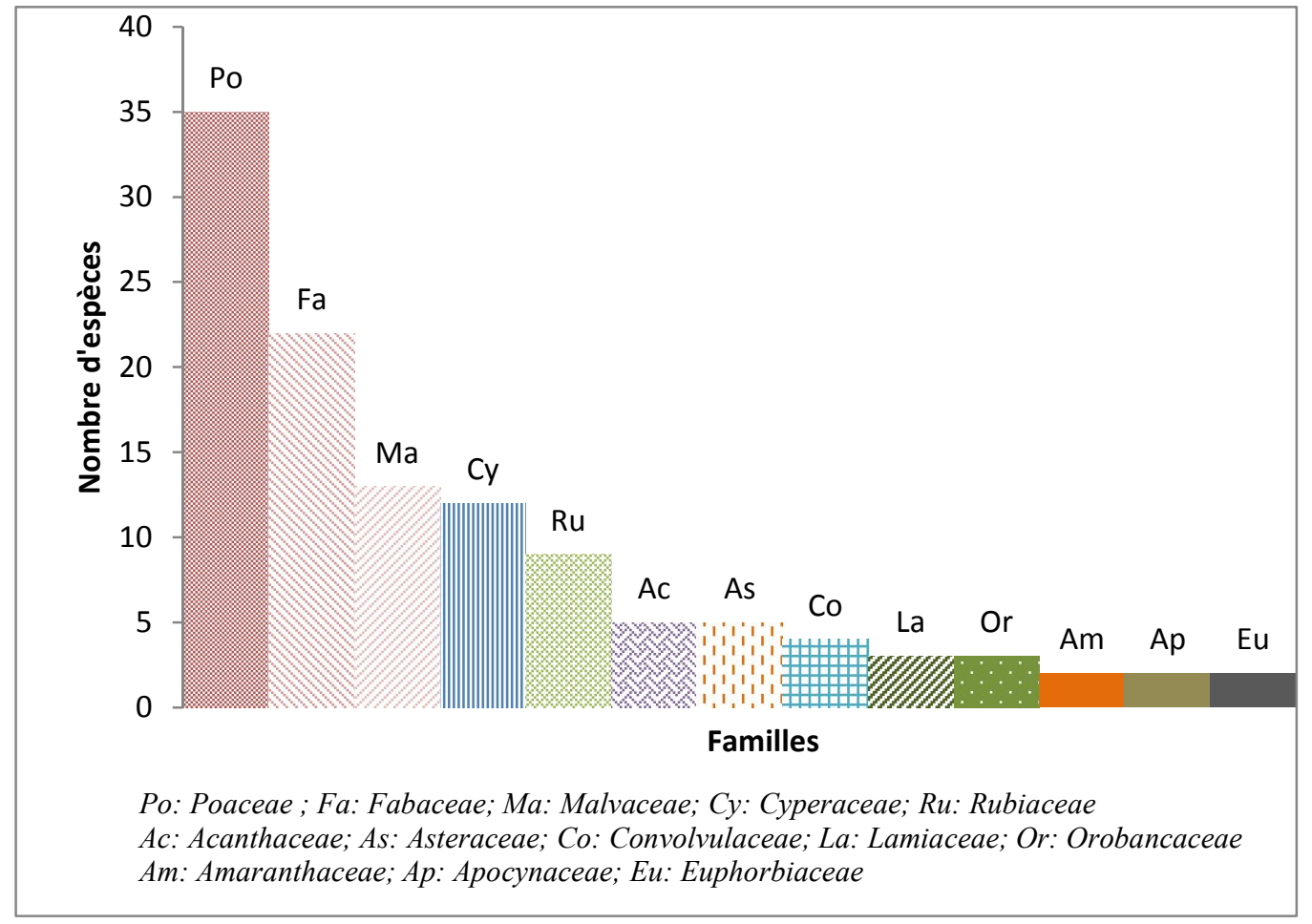

Figure 3 : Spectre des fréquences des familles les plus représentées.

Tableau 1: Diversité floristique des formations végétales des unités géomorphologiques étudiées.

\begin{tabular}{lcccc}
\hline & Plateaux & Zones mixtes & Bas-fonds & Ensemble de relevés \\
\hline Nombre d'espèces & $\mathbf{1 0 0}$ & $\mathbf{8 5}$ & $\mathbf{1 0 0}$ & $\mathbf{1 3 7}$ \\
Nombre de familles & 28 & 23 & 27 & 33 \\
Indice de diversité de Shannon (H') & 4,55 & 4,84 & 5,13 & 5,25 \\
Indice de régularité de Piélou (E) & 0,68 & 0,75 & 0,77 & 0,74 \\
Indice de diversité de Margalef & & & & \\
(MG) & 11,31 & 9,86 & 11,11 & 13,73 \\
\hline
\end{tabular}

Tableau 2 : Taux de similitude entre les listes des espèces des unités géomorphologiques étudiées (exprimés en \%).

\begin{tabular}{lccc}
\hline & Plateaux & Zone mixte & Bas-fond \\
\hline Plateaux & 100 & 57,9 & 54,6 \\
Zones mixtes & 57,9 & 100 & 56,6 \\
Bas-fond & 54,6 & 56,6 & 100 \\
\hline
\end{tabular}


Tableau 3 : Liste des espèces nouvelles de la flore et végétation herbacée du PNM.

\begin{tabular}{|c|c|c|}
\hline Familles & Espèces & Nombre \\
\hline Poaceae & $\begin{array}{l}\text { Andropogon africanus Franch; Beckeropsis uniseta (Ness) K. } \\
\text { Schum, Eragrotis asper (Jacq.) Ness ; } \\
\text { Mnesithea granularis(L) Koning \& Sosef; } \\
\text { Sorghum vulgaris Seed; Sporobolus indicus (L) R. Br. }\end{array}$ & 6 \\
\hline Cyperaceae & $\begin{array}{l}\text { Cyperus esculentus L; Mariscus sp ; Kyllinga squamulata } \\
\text { Thonning \& Valh; Scleria sphaerocarpa (E.A. Rob) Napper. }\end{array}$ & 4 \\
\hline Rubiaceae & $\begin{array}{l}\text { Ageratum conyzö̈des L; Mitracarpus hirtus Forest \& Kim. Starr. } \\
\text { Vernonia ambigua Kotschy \& Peyr ; Launaea cornuta } \text { (Hochst ex }\end{array}$ & 2 \\
\hline Asteraceae & Oliv. \& Hiern) C. Jeffrey. & 2 \\
\hline Fabaceae & Stylosanthes hamata (L.) Taub. & 1 \\
\hline Amaranthaceae & Achyranthes aspera All. & 1 \\
\hline Anthericaceae & Anthericum sp. & 1 \\
\hline Hypodaxidaceae & Curculigo pilosa (Schum \& Thonn.) Engl. & 1 \\
\hline Onagraceae & Ludwigia hyssopifolia (G. Don) Exell & 1 \\
\hline Pedaliaceae & Sesamum angustifolium Thonn. & 1 \\
\hline Phyllanthaceae & Phyllanthus maderaspatensis L. & 1 \\
\hline Polygalaceae & Polygala petitiana A. Rich. & 1 \\
\hline \multirow[t]{2}{*}{ Portulacaceae } & Portulaca quadrifida $\mathrm{L}$. & 1 \\
\hline & Total & 23 \\
\hline
\end{tabular}

\section{DISCUSSION}

Les résultats de cette étude fournissent des informations sur l'état actuel de la végétation herbacée du PNM au Tchad.

La végétation herbacée de la zone du parc étudiée est riche de 137 espèces, reparties en 94 genres et 33 familles. Ce nombre est inférieur à celui obtenu par Saradoum (2012). Cela s'explique par le fait que notre étude ne s'est pas déroulée dans les mêmes zones où Saradoum a réalisé son étude et le nombre de relevés effectués par ce dernier est supérieur aux nôtres.

La superficie totale des relevés effectués est de $60.000 \mathrm{~m}^{2}\left(24 \times 2500 \mathrm{~m}^{2}\right)$ soit $0,46 \%$ de la zone soudanienne du Tchad (46 $\%$ du pays). Malgré la faiblesse de l'aire prospectée, la richesse estimée à 137 espèces est très appréciable, car elle représente 4,98\% $\mathrm{du}$ total de la flore de la zone soudanienne et $3,1 \%$ de la flore nationale. Cette richesse floristique est non exhaustive car 23 espèces herbacées inventoriées lors de notre étude, ne sont pas inventoriées par Saradoum (2012). C'est dire que qu'un travail d'inventaire d'envergure reste à faire pour connaitre la richesse floristique exacte du PNM. Ainsi, les inventaires demeurent le seul moyen pour la valorisation d'un milieu donné (Melom et al., 2015).

Concernant la composition floristique, on constate que la famille des Poaceae est la plus représentée, suivie de celles des Fabaceae, des Malvaceae, des Cyperaceae et des Rubiaceae.

Ces résultats corroborent ceux de Saradoum (2012) qui, dans son étude a trouvé que les espèces de la famille des Poaceae 
étaient les plus nombreuses, suivies de celles des Fabaceae, de Malvaceae, des Cyperaceae et des Rubiaceae.

La forte proportion des Poaceae dans le parc peut s'expliquer par le fait que ces taxons possèdent une très grande possibilité de tallage et une grande vitesse de repousse après broutage lorsque les conditions du milieu sont favorables (Kouassi et al., 2010). Aussi, sont-elles résistantes aux aléas climatiques et sont rarement atteintes par les maladies. Les graminées résistent aux différentes perturbations et elles développent des stratégies leur permettant de se maintenir et de se développer dans un environnement perturbé (Ousseina et al., 2010).

Les familles suivantes: Poaceae, Fabaceae, Malvaceae, Cyperaceae et Rubiaceae renferment 91 espèces soient $66,42 \%$ du total des espèces. A l'exception de la famille des Cyperaceae, elles constituent les groupes d'espèces préférentiellement appétées par les herbivores sauvages (Noupa et al., 2008). Le PNM est un site de pâturage notamment pour les populations animales. La richesse de cet écosystème en espèces de ces cinq familles dominantes justifie son caractère pastoral et son importance pour la conservation des populations animales qui le peuplent.

Il est aussi important de noter que le recouvrement herbacé globalement élevé pour l'ensemble de relevés $(99,73 \%)$ et pour les différentes unités géomorphologiques $(99,30 \%$ pour les plateaux, $99,94 \%$ pour les zones mixtes et $99,95 \%$ pour les bas-fonds) a été évalué durant une année de bonne pluviométrie. Ce recouvrement élevé peut également être lié au statut d'aire protégée de notre site étude.
Les indices de diversité floristiques constituent des critères objectifs pour apprécier la diversité d'une communauté végétale (Ramade, 1994). L'examen de l'indice de diversité $(\mathrm{H})$ et la régularité de Pielou fait apparaître que l'indice de diversité de Shannon pour l'ensemble de la zone d'étude est de 5,25 bits. Cet indice est plus élevé pour la végétation de bas-fonds $(5,13$ bits) que celles des zones mixtes (4,84 bits). Celui de la végétation des zones mixtes est plus élevé que celle des plateaux (4,55 bits). La diversité élevée indique que dans ces différentes unités géomorphologiques la majorité des espèces sont bien représentées, la dominance est faible. Les valeurs élevées de l'équitabilité (supérieur à 0,60 ), indique une bonne équi-répartition des individus entre les différentes espèces, et ce, pour les trois unités géomorphologiques et pour l'ensemble de notre zone d'étude.

Selon les taux de similitude entre les listes des espèces des unités géomorphologiques, il apparait que les relevés des zones mixtes sont similaires à ceux effectues sur les plateaux (57,9\%) et des bas-fonds. Par contre, ceux effectués sur les plateaux paraissent différents de ceux des basfonds $(54,6 \%)$. Les facteurs hydrologiques et pédologiques expliqueraient la distance entre les plateaux et les bas-fonds. Les bas-fonds reçoivent pendant la saison des pluies une grande quantité d'eau de pluies et un apport en matières organiques charriées par les eaux de ruissellement. Ceci justifie sa richesse floristique plus élevée que les plateaux. Ces résultats corroborent ceux de Masharabu (2010) dans le Parc National de la Ruvubu au Burundi. 


\section{Conclusion}

$\mathrm{Au}$ terme de cette étude, l'inventaire floristique réalisé dans le PNM a permis de connaître la composition floristique en herbacées et d'évaluer les indices de diversité floristique. Cette analyse révèle une diversité aussi quantitative que qualitative de l'aire protégée. 137 espèces reparties en 94 genres et 33 familles ont été recensées. Les familles des Poaceae, Fabaceae, Malvaceae, Cyperaceae et Rubiaceae sont les mieux représentées. Toutefois, Les 23 espèces nouvelles pour le PNM mises en évidence témoignent de la faiblesse des investigations sur la flore de cette aire protégée et du travail qui reste à faire afin de révéler ses réelles potentialités floristiques.

Ces résultats constituent une contribution indéniable à l'établissement d'une base de données sur la flore et la végétation du PNM. Ils montrent également que les inventaires restent le seul outil efficace pour l'évaluation et la valorisation des ressources naturelles.

\section{CONFLIT D'INTERETS}

Les auteurs déclarent qu'il n'y a aucun conflit d'intérêts lié à cet article.

\section{CONTRIBUTIONS DES AUTEURS}

L'auteur principal INT a conduit l'étude, collecté, traité les données et a rédigé l'article. Les co-auteurs JLK et JD ont corrigé et finalisé le manuscrit. FR a orienté et supervisé les travaux.

\section{REMERCIEMENTS}

Nous remercions les autorités de la Délégation Régionale de 1'Environnement et des Ressources Halieutiques et le Projet de Conservation et d'Utilisation Durable des
Ressources Naturelles dans le Moyen Chari qui nous ont apporté le soutien nécessaire lors de la collecte des données. Nos sincères remerciements aux collègues enseignantchercheurs de l'Université de Sarh, pour leur critique constructive et apports multidisciplinaires et interinstitutionnelles. Nous adressons aussi notre reconnaissance aux référés anonymes qui ont lu avec attention et fait des observations utiles.

\section{REFERENCES}

Abdelkader Benkhettou, Bel Azouzi, Kadour Djili, Bekhettou Mohamet, Mohamed Zedek, Rachid Saad. 2015. Diversité floristique du massif du Nador en zone steppique (Tiaret, Algérie). European Scientific Journal, 11(21): 1401-419.

Akossoua FK, Yao Adou CY, Ipou JI, Kagoyiré K. 2010. Diversité floristique des zones côtière pâturées de la Côte d'Ivoire : cas du cordon littoral PortBouët-Grand-Bassam (Abidjan). Sciences et Nature, 7(1): 69-86.

Akpo L-E, Grouzis M. 2000. Valeur pastorale des herbages en région soudanienne, le cas des parcours sahéliens du NordSénégal. Tropicultura, 18(1): 1-8.

Akpo L-E, Grouzis M. 2009. Effet des arbres sur la diversité de la végétation herbacée dans les parcours communautaires du Nord-Sénégal (Afrique de l'Ouest). Journal of Agriculture and Environment for International Development, 103(4) : 271-293.

Akpo L-E, Masse D, Grouzis M. 2002. Durée de la jachère et valeur pastorale de la végétation herbacée en zone soudanienne au Sénégal. Rev. Elev.Vet. Pays Trop., 55(4): 275-283. 
ASECNA. 2015. Agence de Sécurité de la Navigation Aérienne en Afrique et à Madagascar, Paramètres météorologiques relatif à la période 2014-2015, 12 p.

Boya Aboh A, Babatoundé S, Oumarou M, Houinato M, Sinsin B. 2012. Valeur pastorale des parcours naturels en zone soudano-guinéenne et stratégie paysanne d'adaptation aux effets de leur invasion par Chromolaena odorata au Bénin. Int. J. Biol. Chem. Sci., 6(4): 1633-1646. DOI : http://dx.doi.org/10.4314/ijbcs. v6i4.22.

Chardonnet B, Boulanodji E. 2010. Plan d'aménagement du Parc National de Manda. Rapport provisoire. Rép. du Tchad, Ministère de l'Environnement et des Ressources Halieutiques /DPNRFC, $175 \mathrm{p}$.

Daouda G, Amy B, Sékouma D, Akpo LE.2012. Qualité pastorale des ressources herbagère de la réserve de biosphère de Ferlo (Nord-Sénégal). Int. J. Biol. Chem. Sci., 6(1): 186-201. DOI: $\quad h t t p / /: d x . d o i . o r g / 10.4314 /$ ijbcs. v6i1.17.

Deguemo DD, Tientcheu MA, Mbaïti D, Saradoum G, Pinta JY. 2017. Importance écologique et potentialité apicole à la périphérie du Parc National de Manda en zone soudanienne du Moyen Chari (Tchad). International Journal of Environment Studies, 74(3), 443-457. DOI : http:// dx.doi.org/10. 1080/00207233.2017.1294424.

Dibi HN'd, Yao CYA, Kouakou EN, Moussa K, Yao CS. 2008. Analyse de la diversité floristique du parc national de Marahoué, Centre-Ouest de la Côte d'Ivoire. Afrique Sciences, 04(3) 552-579.
Goy S, Aly D, Madiara N F, Aliou G. 2012. Characterization of the herbaceous vegetation of the National Park of Manda in Chad. International Journal of Sciences and Advanced Technology. 2(6): 1-10.

Goy S. 2012. Etude phytosociologique et diagnostic faunique du parc national de Manda au Tchad; éléments pour un aménagement. Thèse de doctorat. Université Cheikh Anta Diop de Dakar. $179 \mathrm{p}$.

Ismaïla Toko, Brice Sinsin. 2011. Facteurs déterminant la variabilité spatiale de la biomasse herbacée dans la zone soudano-guinéenne du Bénin. Int. J. Biol. Chem. Sci., 5(3) : 930-943. DOI : http://dx.doi.org/10.4314/ijbcs.v5i3.7218 0 .

Jean-Peirre Tiendrébego, Dr Jean-Pierre Sorg. 1997. Etude de la capacité de Charge de la forêt classée de Gonsé. MEE/SG/DGEF/GTZ, Ouagadougou (Burkina Faso) : GTZ, 1997.

Kiema A, Sawadogo I, Tinrmegson O, Nianogo AJ. 2012. Stratégies d'exploitation du fourrage par les éleveurs de la zone sahélienne du Burkina Faso. Int. J. Biol. Chem. Sci., 6(4): 1492-1505. DOI : hptt//dx.doi.org/ 10.4314 ijibcs. v6i4.8

Kolmagne MN. 2000. Etude du peuplement de faune et de flore dans le parc national de Manda. Etats de surface : - Transects dans le parc, - Elément de typologie des espèces inventoriées, - Axes d'intervention comme suite à cette étude. Rapport consultatif. APRODEPIT, CARE-TCHAD. Ndjamena. 72 pages.

Kossi A, Bellefontaine R, Kokou K. 2009. Les forêts claires du Parc national Oti-Kéran 
au Nord-Togo : structure, dynamique et impacts des modifications climatiques récentes. Sècheresse, 20(1): 834-856. DOI : 10.1684/sec.2009.0217.6.

Kouassi AF, Koffi KJ, N'Goran KS Béranger, IJ. 2014. Potentiel de production fourragère d'une zone pâturée menacée de destruction: cas du cordon littoral Port-Bouët et Grand-Bassam. Journal of Applied Bioscience, 82: 7403-7410. DOI: $\quad$ http://dx.doi.org/10.4314/jab. v82i1.13

Melom S, Mbayngone E, Béchir AB, Ratnab N, Mapongmetsem PM. 2015. Caractéristiques floristiue et écologique des formations végétales de Massenya au Tchad (Afrique centrale). Journal of Animal \& Plan Sciences, 25(1): 37993813.

MERH (Ministère de 1'Environnement et des Ressources halieutiques). 2014. $5^{\text {eme }}$ Rapport National sur la biodiversité au Tchad. MERH-PNUE-FEM, 65 p.

Minda MS, Diallo MD, Sarr PS, Ndiaye O, Touroumgaye G, Goy S, Guisse A. 2016. Diversité floristique des peuplements herbacés des trois types de tracé de la Grande Muraille Verte du Tchad. Annales des Sciences Agronomiques 20(2): 1-12.

Minda M-S, Ousmane N, Mariama DD, Saradoum G, Ally D, Alliou G. 2015. Caractérisation des peuplements ligneux sur le tracé de la Grande Muraille Verte au Tchad. Int. J. Biol. Chem. Sci., 9(5): 2617-2627. DOI : http://dx.doi.org/10. 4314/ijbcs.v9i5.31.

Ngarnougber C, Tonalta FN, Adamou I. 2017. Caractérisation des ligneux de la savane sahélienne à Acacia senegal (L) Will dans la région du Guéra, Tchad.
International Journal of Applied Research, 3(4):600:606. DOI : 10.4000/vertigo.17282.28.

Noupa P, Nkongmeneck B-A. 2008. Evaluation de la diversité floristique de six clairières sur le massif forestier Boumba-Bek et Nki, Sud-Est Cameroun. Int. J. Biol. Chem. Sci., 2(4): 529-548. DOI : $\quad$ http://dx.doi.org/10.4314/ijbcs. v2i4.3976․․

Nshimba SwMH. 2007. Etude floristique, écologique et phytosociologique des forêts de l'île Mbiye, Kisangani. R.D. Congo. Thèse de doctorat. Université Libre de Bruxelles, Laboratoire de Botanique Systématique et de Phytosociologie. Belgique. 420 pages.

Onana J, Mvondo Awono JP, Abba D, Ottou JFB. 2008. Application de la méthode de points quadrats alignés au suivi de la dynamique des pâturages soudanosaheliens du Cameroun. Int. J. Biol. Chem. Sci., 2(2): 224-229. DOI : http://dx.doi.org/10.4314/ijbcs.v2i2.3974 1.

Ousseina S, Soumana D, Ali Z, Ricardo F. 2010. Analyse du peuplement herbacé de la station expérimentale de Toukounous (Niger): composition floristique et valeur pastorale. Sècheresse, 21(1): 154160. DOI : $10.1684 / \mathrm{sec} .2010 .0243$.

Ouya B. 2010. Conservation et utilisation durable de la biodiversité autour et dans les aires protégées du Sud-Est du Tchad : cas du Parc de Manda et de la Foret de Djoli-Kera. Usages et risques, quelles stratégies pour la revalorisation et le développement local? Thèses de doctorat de Géographie et Aménagement des espaces. Université Paul-Valery, Montpellier, 296 p. 
Palayer P. 1977. Lexique de plantes du pays Sar: plantes spontanées et cultivées. Tome II- noms scientifiques-noms Sar avec indications d'utilisation. Ronéo. Centre d'Études Linguistiques. Collège Charles Lwanga. Sarh, 77p.

Pias J. 1964. Les sols du Tchad. VIIIème Congrès International de la Science du Sol, Bucarest- Roumanie. Comptes rendus, 145-151.

Rachad KFMA, Odjoubere J, Tente ABH, Sinsin AB. 2014. Caractérisation floristique et analyse des formes de pression sur les forêts sacrées ou communautaires de la Basse Vallée de l'Ouémé au Sud-Est du Bénin. Afrique Science, 10(2) (2014) 243-257.

Tatien Masharabu, Marie José Bigendako, Jean Lejoly, Jacques NKengurutse, Naussicaa Noret, Elias Bizuru, Jean Bogaert. 2010. Etude analytique de la flore et de la végétation du Parc National de la Ruvubu, Burundi. Int. J. Biol. Chem. Sci., 4(4): 834-856.

Tiebre M-S, Ouatara D, Kpangui KB, Kouassi DF, N'guessan KE. 2016. Diversité floristique de la région de Foungbesso en zone de transition Forêt-savane à l'ouest de la Côte d'Ivoire.

Int. J. Biol. Chem. Sci., 10(3) : 1007-1016. DOI: hptt://dx.doi.org/10.4314/ijbcs.v10i3.8.

Yameogo G, Kiema A, Yelemou B, Ouedraogo L. 2013. Caractéristiques des ressources fourragères herbacées des pâturages naturels du territoire de Vipalogo (Burkina Faso). Int. J. Biol. Chem. Sci., 7(5) : 2078-2091. DOI : http://dx.doi.org/10.4314/ijbcs.v7i5.25.

Yemye LW. 2010. Rapport de collecte des données nationales - Tchad. Aires Protégées Résilientes au Changement Climatiques, PARCC Afrique de 1'Ouest. UNET, UICN, GEF, 38 p.

Yoka J, Loumeto JJ, Vouidibio J, Amiaud B, Eperon D. 2010. Influence du sol sur la répartition et la production de phytomasse de savanes de la Cuvette congolaise (République du Congo). GeoEco-Trop., 34: 63-74.

Yoka J, Loumeto JN, Djego J, Vouidibio J, Epron D. 2013. Evaluation de la diversité floristique en herbacées des savanes de la cuvette congolaise (République du Congo). Afrique Science, 09(2): 110-123. 